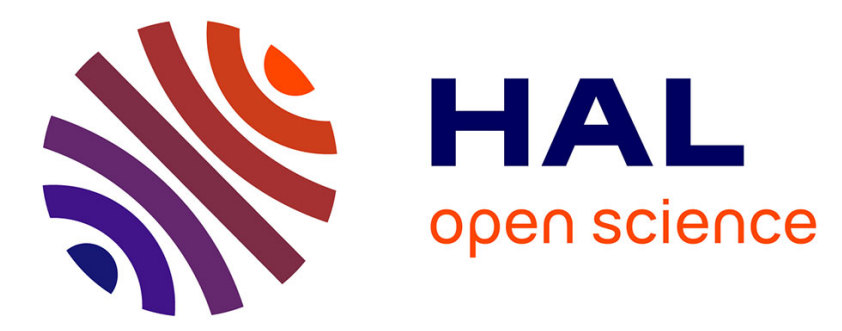

\title{
Solvent free hydrostannation and Stille reactions using ionic liquid supported organotin reagents
} Djibril Faye, Mykhailo Vybornyi, Fabien Boeda, Stéphanie Legoupy

\section{To cite this version:}

Djibril Faye, Mykhailo Vybornyi, Fabien Boeda, Stéphanie Legoupy. Solvent free hydrostannation and Stille reactions using ionic liquid supported organotin reagents. Tetrahedron, 2013, 69, pp.5421-5425. 10.1016/j.tet.2013.04.116 . hal-03344624

\section{HAL Id: hal-03344624 \\ https://univ-angers.hal.science/hal-03344624}

Submitted on 15 Sep 2021

HAL is a multi-disciplinary open access archive for the deposit and dissemination of scientific research documents, whether they are published or not. The documents may come from teaching and research institutions in France or abroad, or from public or private research centers.
L'archive ouverte pluridisciplinaire HAL, est destinée au dépôt et à la diffusion de documents scientifiques de niveau recherche, publiés ou non, émanant des établissements d'enseignement et de recherche français ou étrangers, des laboratoires publics ou privés. 

Not for reproduction, distribution or commercial use.

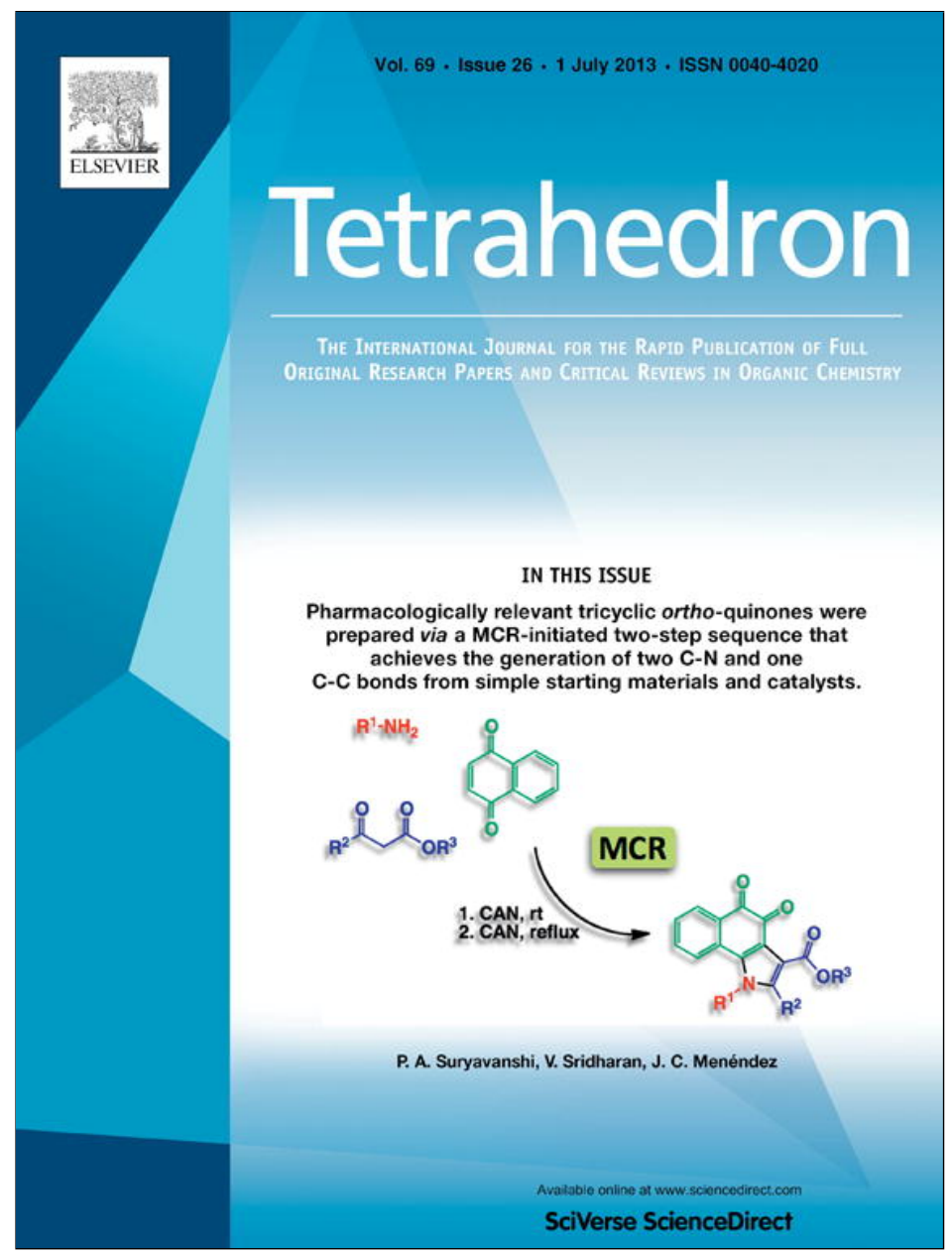

This article appeared in a journal published by Elsevier. The attached copy is furnished to the author for internal non-commercial research and education use, including for instruction at the authors institution and sharing with colleagues.

Other uses, including reproduction and distribution, or selling or licensing copies, or posting to personal, institutional or third party websites are prohibited.

In most cases authors are permitted to post their version of the article (e.g. in Word or Tex form) to their personal website or institutional repository. Authors requiring further information regarding Elsevier's archiving and manuscript policies are encouraged to visit:

http://www.elsevier.com/authorsrights 


\title{
Solvent free hydrostannation and Stille reactions using ionic liquid supported organotin reagents
}

\author{
Djibril Faye ${ }^{\mathrm{a}}$, Mykhailo Vybornyi ${ }^{\mathrm{a}}$, Fabien Boeda ${ }^{\mathrm{b}}$, Stéphanie Legoupy ${ }^{\mathrm{a}, *}$ \\ a LUNAM Université, Université d'Angers, CNRS UMR 6200, Laboratoire MOLTECH-Anjou, 2 bd Lavoisier, 49045 Angers, France \\ b LUNAM Université, Université du Maine, CNRS UMR 6283, IMMM - Institut des Molécules et Matériaux du Mans, Av O. Messiaen, 72085 Le Mans, \\ France
}

\section{A R T I C L E I N F O}

Article history:

Received 13 February 2013

Received in revised form 22 April 2013

Accepted 24 April 2013

Available online 1 May 2013

\section{Keywords:}

Hydrostannation

Alkynes

Palladium

Stille reaction

Ionic liquid

\begin{abstract}
A B S T R A C T
Hydrostannation reactions were performed cleanly using ionic liquid supported organotin reagents. These green reducing agents were used both under free radical and palladium-catalyzed conditions. One of the new ionic liquid supported organotin reagents so obtained was evaluated successfully in Stille cross-coupling reactions to give aryl-substituted allylic alcohols in solvent free conditions.
\end{abstract}

(c) 2013 Elsevier Ltd. All rights reserved.

\section{Introduction}

During the past decades, many versatile and efficient synthetic methods using organotin reagents (Stille coupling, radical reactions, allylation reactions...) have emerged as powerful tools for organic chemists. However, despite such impressive synthetic potential, reactions involving organotin compounds display drawbacks, such as pollution of products by tin salts and difficulties of separation at the end of the synthesis. As a result, ongoing efforts have been devoted to overcome these problems by using solid phase synthetic methods, ${ }^{1}$ phosphonium grafted organotins, ${ }^{2}$ catalytic Stille couplings, ${ }^{3}$ and other modified organotin reagents. ${ }^{4}$ As a part of our ongoing research program on the discovery of potentialities of TSILs (task specific ionic liquids), ${ }^{5}$ we became interested in investigating the use of organotin reagents supported on ionic liquids for hydrostannation reaction.

Vinylstannanes are useful intermediates for organic synthesis. Among the various methods to obtain vinylstannanes, the most studied and frequently used one is the addition of a tin hydride species to an alkyne. ${ }^{6}$ Following this strategy, Cai et al. ${ }^{7}$ described palladium-catalyzed hydrostannation reactions of various alkynes in ionic liquids. Recently, we reported the synthesis of organotin

\footnotetext{
* Corresponding author. Tel.: +332 41735375; fax: +33 2 41735405; e-mail address: stephanie.legoupy@univ-angers.fr (S. Legoupy).
}

reagents supported on ionic liquid bearing a vinyl moiety. These compounds were obtained after reaction of a Grignard reagent with an organotin chloride derivative. ${ }^{5 e}$ As it is somehow difficult to prepare functionalized vinyl Grignard reagents, we report herein the synthesis of a series of alkenes supported on ionic liquids using hydrostannation reactions. A preliminary study concerning their use in Stille cross-coupling reactions is also described in this work.

\section{Results and discussion}

As an initial assay, hydrostannation reactions under free radical conditions were investigated using ionic liquid-supported organotin hydride 2 . This compound was obtained by reduction of $\mathbf{1}^{5 \mathrm{C}}$ in presence of sodium borohydride and methanol (Scheme 1).

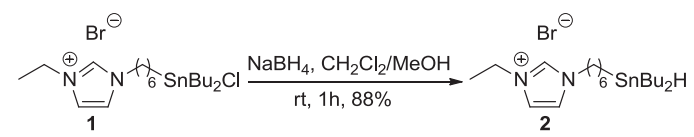

Scheme 1. Preparation of organotin hydride supported on ionic liquid.

In a general manner, ionic liquids were isolated with moderate yields (40-55\%) as highlighted in Table 1 . The hydrostannation of phenyl acetylene (Table 1, entry 1 ) proceeded with no selectivity. When using propargyl alcohol as substrate (Table 1, entry 2 ), selectivity was improved in favor of trans-isomer. Unfortunately, 
Table 1

Hydrostannation reactions under free radical conditions

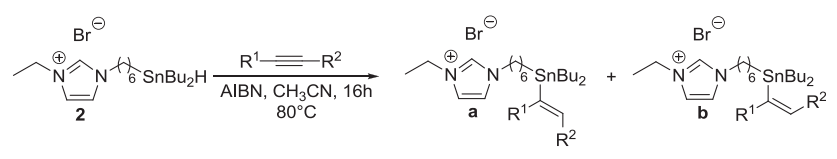

\begin{tabular}{lllll}
\hline Entry & $\mathrm{R}^{1}$ & Ratio $\mathbf{a} / \mathbf{b}^{\mathrm{a}}$ & Yield (\%) \\
\hline & & $\mathbf{3 a} / \mathbf{3 b} 50: 50$ & 40 \\
& & $\mathbf{4 a} / \mathbf{4 b} 82: 18$ & 42 \\
& & $\mathbf{5 a} / \mathbf{5 b} 2: 98$ & 48 \\
\hline
\end{tabular}

${ }^{\mathrm{a}}$ Determined by ${ }^{1} \mathrm{H}$ NMR.

isomers $\mathbf{3 a} / \mathbf{3 b}$ and $\mathbf{4 a} / \mathbf{4 b}$ could not be separated by silica gel column chromatography. With a symmetrical alkyne (Table 1 , entry 3 ) and a sterically demanding alkyne (Table 1 , entry 4 ), one isomer was mainly obtained, $(Z)$ - and (E)-isomer, respectively.

The reactivity of tin hydride $\mathbf{2}$ has been evaluated in palladiumcatalyzed hydrostannation reactions with different precatalysts (3 mol \%) under solvent free conditions by using 3-methyl-3hydroxybut-1-yne 7 as a benchmark substrate. Results are summarized in Table 2 .

Table 2

Precatalyst effect on palladium-catalyzed hydrostannation reactions

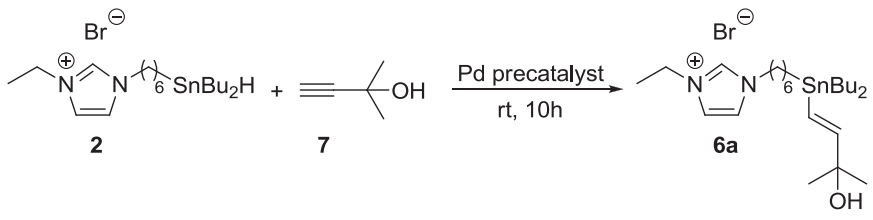

\begin{tabular}{lll}
\hline Entry & Precatalyst & Yield (\%) \\
\hline 1 & {$[\mathrm{Pd} / \mathrm{C}]$} & - \\
2 & {$\left[\mathrm{Pd}(\mathrm{OAc})_{2}\right] / 2 \mathrm{PPh}_{3}$} & 18 \\
3 & {$\left[\mathrm{PdCl}{ }_{2}\right] / 2 \mathrm{PPh}_{3}$} & 21 \\
4 & {$\left[{\left.\mathrm{Pd}\left(\mathrm{PPh}_{3}\right)_{4}\right]}_{78}\right.$} & 85 \\
5 & {$\left[\mathrm{PdCl}_{2}\left(\mathrm{PPh}_{3}\right)_{2}\right]$} & 80 \\
6 & {$\left[\mathrm{PdCl}_{2}\left(\mathrm{PPh}_{3}\right)_{2}\right]^{\mathrm{a}}$} & 80 \\
\hline
\end{tabular}

a THF was used as co-solvent.

No conversion was observed with Pd/C (Table 2, entry 1 ). When using $\left[\mathrm{Pd}(\mathrm{OAc})_{2}\right]$ or $\left[\mathrm{PdCl}_{2}\right]$ both with 2 equiv of triphenylphosphine (Table 2 , entries 2 and 3 ), the reaction proceeded with only $18-21 \%$ conversion. Then, the use of $\left[\mathrm{Pd}\left(\mathrm{PPh}_{3}\right)_{4}\right]$ allowed the formation of compound $\mathbf{6 a}$ with $78 \%$ yield (Table 2, entry 4 ). Interestingly, the highest conversion (85\%) was observed when using $\left[\mathrm{PdCl}_{2}\left(\mathrm{PPh}_{3}\right)_{2}\right]$ as precatalyst (Table 2 , entry 5$)$. To further optimize reaction conditions, the influence of a co-solvent was investigated (Table 2, entry 6). Noteworthy, the use of THF as additive did not improve the yield.

With these optimized conditions in hand, we decided to investigate the scope of our catalytic system. Indeed, the hydrostannation of a series of alkynes was studied and the results are depicted in Table 3. We have shown that the palladium-catalyzed hydrostannation reaction could be done with in situ tin hydride
Table 3

Palladium-catalyzed hydrostannation reactions

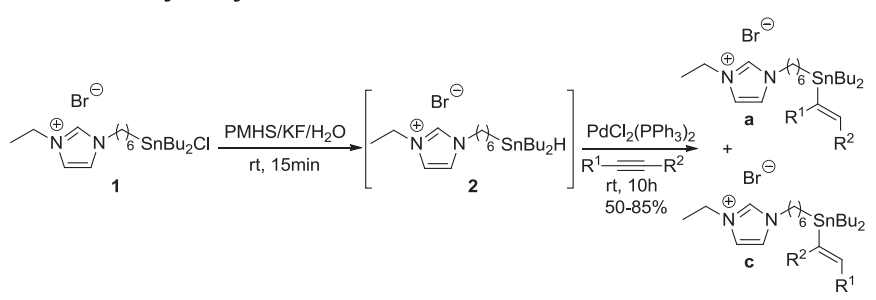

\begin{tabular}{|c|c|c|c|c|}
\hline Entry & $\mathrm{R}^{1}$ & $\mathrm{R}^{2}$ & Ratio $\mathbf{a} / \mathbf{c}^{\mathrm{a}}$ & Yield (\%) \\
\hline 1 & $\mathrm{H}$ & & $\mathbf{3 a} / \mathbf{3 c} 40: 60$ & 65 \\
\hline 2 & $\mathrm{H}$ & & $\mathbf{4 a} / \mathbf{4 c} 12: 88$ & 72 \\
\hline 3 & & & $5 a$ & 75 \\
\hline 4 & $\mathrm{H}$ & & $\mathbf{6 a / 6 c} 95: 5$ & 85 \\
\hline 5 & $\mathrm{H}$ & & 7a/7c 5:95 & 77 \\
\hline 6 & $\mathrm{H}$ & & $\mathbf{8 a} / \mathbf{8 c} 62: 38$ & 50 \\
\hline 7 & $\mathrm{H}$ & & 9a/9c $40: 60$ & 52 \\
\hline 8 & $\mathrm{H}$ & & 10a/10c $95: 5$ & 76 \\
\hline
\end{tabular}

${ }^{\mathrm{a}}$ Determined by ${ }^{1} \mathrm{H}$ NMR.

generation. This species was generated in situ with hypercoordinate polymethylhydroxysilane ${ }^{3}$ (PMHS+fluoride source).

Using our optimized conditions, hydrostannation products were obtained in moderate to good yields following mild reaction conditions. It should be noted that good regioselectivities were observed with sterically demanding alkynes (Table 3 , entries 4 and 8 ) leading mainly to one isomer in case of $\mathbf{6 a}$ and $\mathbf{1 0 a}$.

The influence of the ionic liquid's counter anion on the hydrostannation reaction has been investigated (Scheme 2). It has been shown that this parameter strongly influences the yield of the reaction. Indeed, anionic metathesis reactions between bromide and tetrafluoroborate or hexafluoroantimonate anions have to be achieved after hydrostannation reaction, otherwise low yields are obtained (30 and $15 \%$, respectively). This phenomenon is probably connected to the strength of cation-anion interaction ${ }^{8}$ $\mathrm{SbF}_{6}{ }^{-}>\mathrm{BF}_{4}^{-}>\mathrm{Br}^{-}$, also confirmed by the ${ }^{1} \mathrm{H}$ chemical shifts of the proton at $\mathrm{C} 2$ position of imidazolium ring.

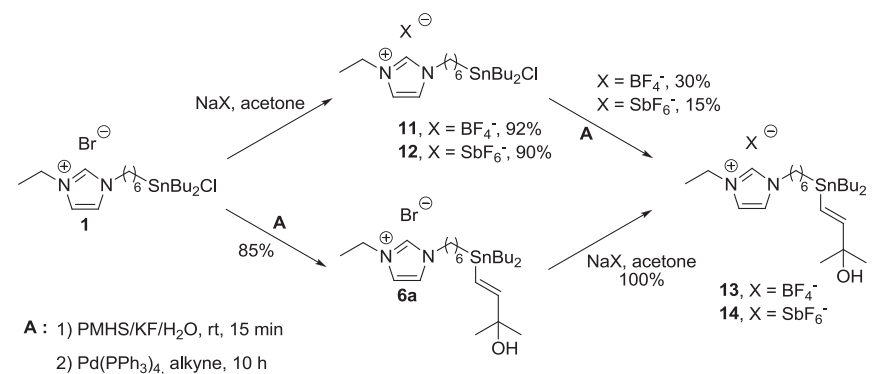

Scheme 2. Influence of ionic liquid's counter anion on hydrostannation. 
Finally, we were interested in the reactivity of compound $\mathbf{6 a}$ in Stille cross-coupling reactions. Conditions previously optimized with brominated substrates ${ }^{5 e}\left(130{ }^{\circ} \mathrm{C}\right.$ and $\left[\mathrm{Pd}\left(\mathrm{PPh}_{3}\right)_{4}\right]$ as precatalyst) were applied (Table 4 ).

\section{Table 4}

Stille cross-coupling reactions
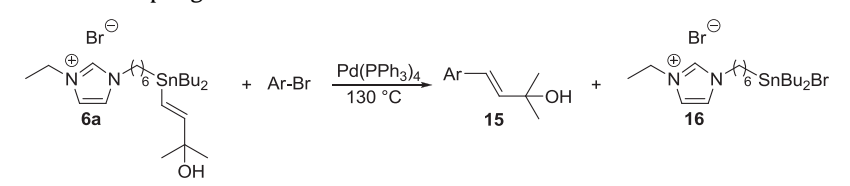

\begin{tabular}{lll}
\hline Entry & Aryl bromide & Yield (\%) \\
\hline 1 & 15a 81 \\
& & 15b 84 \\
\hline
\end{tabular}

At the end of the Stille reaction, desired products $15 \mathbf{a}-\mathbf{d}$ were isolated with good yields (Table 4, entries $1-4$ ) by simple extraction with diethylether. As shown in our previous work, the organotin supported ionic liquid reagent $\mathbf{1 6}$ could be recycled without loss of reactivity. ${ }^{5 e}$

\section{Conclusion}

In summary, hydrostannation reactions using ionic liquidsupported organotin reagents were studied. Both free radical and palladium-catalyzed conditions have been tested and allowed the synthesis of eight novel ionic liquid-supported organotin reagents. The in situ tin hydride generation directly followed by palladiumcatalyzed hydrostannation reaction led to a straightforward access to functionalized ionic liquid-supported organotin reagents. The scope of ionic liquid $\mathbf{6 a}$ was evaluated for several Stille crosscoupling reactions. Overall, the methodologies disclosed in this paper offer clean synthetic methods to give aryl-substituted allylic alcohols: no solvent used in both hydrostannation and Stille reactions, ease of purification, no tin contamination. Further applications of organotin reagents supported on ionic liquids are currently underway in our laboratories and will be disclosed shortly.

\section{Experimental section}

\subsection{General}

Commercially available reagents and solvents were purified and dried, when necessary, by standard methods prior to use. ${ }^{1} \mathrm{H}$ (300 MHz), ${ }^{13} \mathrm{C}(75 \mathrm{MHz})$ NMR spectra were recorded on a Bruker Avance 300 spectrometer. The compounds studied were measured in $\mathrm{CDCl}_{3}$ and ${ }^{1} \mathrm{H}$ and ${ }^{13} \mathrm{C}$ chemical shifts, reported in parts per million, were referred to the central signal of the solvent. ${ }^{13} \mathrm{C}$ NMR spectra were recorded with complete proton decoupling. The ${ }^{119} \mathrm{Sn}$ NMR spectra were recorded on a Bruker Avance 400 spectrometer (149 MHz) and chemical shifts were referred to external tetramethylstannane. High resolution mass spectra measurements were recorded on Waters-Micromass GCT Premier spectrometers. Analytical thin layer chromatography was performed on pre-coated silica gel 60-F254 plates. The following compounds have been previously described: $1,{ }^{5 \mathrm{c}} \mathbf{1 5 a},{ }^{9} \mathbf{1 5 b},{ }^{10} \mathbf{1 5 c},{ }^{11} \mathbf{1 5 d}{ }^{12}$ Compounds $3, \mathbf{4}$, $\mathbf{8}$, and $\mathbf{9}$ have been isolated as a mixture of regioisomers (NMR data is provided only for the major product).

4.1.1. General procedure for hydrostannation under radical conditions. To tin hydride $2(0.494 \mathrm{mg}, 1 \mathrm{mmol})$ in $\mathrm{CH}_{3} \mathrm{CN}(2 \mathrm{~mL})$, the alkyne substrate $(1 \mathrm{mmol})$ and AIBN (3 mg, $0.019 \mathrm{mmol}$, 0.01 equiv) were added. After $10 \mathrm{~h}$ of reaction at reflux, the mixture was cooled to room temperature and concentrated under reduced pressure. The residue was purified by silica gel flash column chromatography $\left(\mathrm{CH}_{2} \mathrm{Cl}_{2} / \mathrm{MeOH} 100 / 0\right.$ to $\left.85 / 15\right)$ to afford ionic liquids 3-6.

4.1.2. General procedure for precatalyst effect on palladium catalyzed hydrostannation. To tin hydride $2(0.494 \mathrm{mg}, 1 \mathrm{mmol})$, the alkyne substrate $(1 \mathrm{mmol})$ and palladium precatalyst $(0.03 \mathrm{mmol})$ were added. After $10 \mathrm{~h}$ of reaction at room temperature, the solution was purified by silica gel flash column chromatography $\left(\mathrm{CH}_{2} \mathrm{Cl}_{2} / \mathrm{MeOH}\right.$ $100 / 0$ to $85 / 15$ ) to afford ionic liquids $6 \mathbf{a}$.

4.1.3. General procedure for palladium catalyzed hydrostannation with in situ tin hydride generation. To tin chloride $1(528 \mathrm{mg}$, $1 \mathrm{mmol})$ were added $3 \mathrm{M} \mathrm{KF}_{\mathrm{aq}}$ solution $(0.83 \mathrm{~mL}, 2.5 \mathrm{mmol})$ and PMHS (0.15 mL, $2.5 \mathrm{mmol})$. The resulting solution was allowed to stir $15 \mathrm{mn}$ at room temperature, then the alkyne substrate $(1 \mathrm{mmol})$ and $\mathrm{PdCl}_{2}\left(\mathrm{PPh}_{3}\right)_{2}(21 \mathrm{mg}, 0.03 \mathrm{mmol})$ were added. After $10 \mathrm{~h}$ of reaction at room temperature, the solution was purified by silica gel flash column chromatography $\left(\mathrm{CH}_{2} \mathrm{Cl}_{2} / \mathrm{MeOH} 100 / 0\right.$ to $\left.85 / 15\right)$ to afford ionic liquid 3-10.

4.1.4. General procedure for anion metathesis. A solution of ionic liquid 1 or $\mathbf{6 a}(1.92 \mathrm{mmol})$ in acetone $(25 \mathrm{~mL})$ was stirred with corresponding salt (1.5 equiv) at room temperature for $24 \mathrm{~h}$. The reaction mixture was filtered off to remove the insoluble materials. The acetone was evaporated in vacuo and the product was dried under reduced pressure to give ionic liquid 11-14.

4.1.5. General procedure for Stille cross-coupling reactions. To a solution of ionic liquid $\mathbf{6 a}(0.173 \mathrm{mmol}), \mathrm{Pd}\left(\mathrm{PPh}_{3}\right)_{4}(3 \mathrm{~mol} \%)$ and aryl bromide $(0.260 \mathrm{mmol})$ were added. The mixture was stirred for $24 \mathrm{~h}$ at $130^{\circ} \mathrm{C}$. The solution was extracted with diethylether and concentrated under reduced pressure. The residue was purified by silica gel column chromatography $\left(\mathrm{CH}_{2} \mathrm{Cl}_{2} / \mathrm{MeOH} 100 / 0\right.$ to $\left.97 / 3\right)$ to afford aryl-substituted allylic alcohols $\mathbf{1 5 a} \mathbf{a}-\mathbf{d}$.

4.1.6. 1-((Dibutylstannyl)hexyl)-3-ethyl-1H-imidazol-3-ium bromide (2). To a suspension of $\mathrm{NaBH}_{4}(28 \mathrm{mg}, 0.75 \mathrm{mmol})$ in $\mathrm{CH}_{2} \mathrm{Cl}_{2}(5 \mathrm{~mL})$, a solution of $\mathbf{1}(132 \mathrm{mg}, 0.25 \mathrm{mmol})$ in mixture of $\mathrm{CH}_{2} \mathrm{Cl}_{2}(5 \mathrm{~mL})$ and $\mathrm{MeOH}(20 \mu \mathrm{L})$ was added slowly at $0{ }^{\circ} \mathrm{C}$. The resulting mixture was stirred for $1 \mathrm{~h}$ at room temperature. Water $(10 \mathrm{~mL})$ was added to the reaction mixture, and the aqueous phase was extracted with $\mathrm{CH}_{2} \mathrm{Cl}_{2}$ $(3 \times 10 \mathrm{~mL})$. The combined organic phases were then dried over $\mathrm{MgSO}_{4}$, filtered, and concentrated under reduced pressure to yield 2 (109 $\mathrm{mg}, 88 \%)$ as a yellow viscous oil.

${ }^{1} \mathrm{H}$ NMR $\left(\mathrm{CDCl}_{3}\right): \delta 9.97\left(\mathrm{~s}, 1 \mathrm{H}, \mathrm{CH}_{\text {imidazolium }}\right), 7.50(\mathrm{~d}, J=1.9 \mathrm{~Hz}$, $\left.1 \mathrm{H}, \mathrm{CH}_{\text {imidazolium }}\right), 7.35\left(\mathrm{~d}, J=1.9 \mathrm{~Hz}, 1 \mathrm{H}, \mathrm{CH}_{\text {imidazolium }}\right), 4.58(\mathrm{~m}, 1 \mathrm{H}$, $\mathrm{Sn}-\mathrm{H}$ ), 4.36 (q, J=7.5 Hz, 2H, N- $\left.\mathrm{CH}_{2}-\mathrm{CH}_{3}\right), 4.26(\mathrm{t}, J=7.5 \mathrm{~Hz}, 2 \mathrm{H}$, $\left.\mathrm{N}-\mathrm{CH}_{2}-\mathrm{CH}_{2}\right), 1.86\left(\mathrm{~m}, 2 \mathrm{H}, \mathrm{N}-\mathrm{CH}_{2}-\mathrm{CH}_{2}\right), 1.55(\mathrm{t}, J=7.5 \mathrm{~Hz}, 3 \mathrm{H}$, $\left.\mathrm{N}-\mathrm{CH}_{2}-\mathrm{CH}_{3}\right), 1.21-1.49\left(\mathrm{~m}, 14 \mathrm{H}, \mathrm{H}_{\text {alkyl }}\right), 0.80-0.90\left(\mathrm{~m}, 12 \mathrm{H}, \mathrm{H}_{\text {alkyl }}\right)$. ${ }^{13} \mathrm{C}$ NMR $\left(\mathrm{CDCl}_{3}\right)$ : $\delta 136.8,121.8,121.7,53.4,49.9,45.1,33.3,30.2$, 29.7, 27.3, 26.9, 25.7, 15.5, 13.6, 8.1. ${ }^{119} \mathrm{Sn} \mathrm{NMR}\left(\mathrm{CDCl}_{3}\right): \delta-88.4$. HRMS calcd for $\mathrm{C}_{19} \mathrm{H}_{39} \mathrm{~N}_{2} \mathrm{Sn} 415.2135[\mathrm{M}-\mathrm{Br}]^{+}$; found 415.2132 . 
4.1.7. 1-(6-(Dibutyl(styryl)stannyl)hexyl)-3-ethyl-1H-imidazol-3ium bromide (3c). ${ }^{1} \mathrm{H}$ NMR $\left(\mathrm{CDCl}_{3}\right): \delta 10.49\left(\mathrm{~s}, 1 \mathrm{H}, \mathrm{CH}_{\text {imidazolium }}\right)$, 7.56-7.40 (m, 2H, CH $\left.\mathrm{CH}_{\text {imidazolium }}\right), 7.37-7.24\left(\mathrm{~m}, 3 \mathrm{H}, \mathrm{CH}_{\text {arom }}\right)$, 7.23-7.13 (m, $\left.2 \mathrm{H}, \mathrm{CH}_{\text {arom }}\right), 6.04\left(\mathrm{~d}, J=2.5 \mathrm{~Hz}, 1 \mathrm{H}, \mathrm{CH}_{\text {alkene }}\right), 5.42(\mathrm{~d}$, $\left.J=2.5 \mathrm{~Hz}, 1 \mathrm{H}, \mathrm{CH}_{\text {alkene }}\right), 4.44\left(\mathrm{q}, J=7.3 \mathrm{~Hz}, 2 \mathrm{H}, \mathrm{N}-\mathrm{CH}_{2}-\mathrm{CH}_{3}\right), 4.30$ (t, $\left.J=7.2 \mathrm{~Hz}, 2 \mathrm{H}, \mathrm{N}-\mathrm{CH}_{2}-\mathrm{CH}_{2}\right), 1.91-1.79\left(\mathrm{~m}, 2 \mathrm{H}, \mathrm{N}-\mathrm{CH}_{2}-\mathrm{CH}_{2}\right), 1.61(\mathrm{t}$ $\left.J=7.3 \mathrm{~Hz}, 3 \mathrm{H}, \mathrm{N}-\mathrm{CH}_{2}-\mathrm{CH}_{3}\right), 1.55-1.24\left(\mathrm{~m}, 14 \mathrm{H}, \mathrm{H}_{\mathrm{alkyl}}\right), 1.05-0.78(\mathrm{~m}$, $\left.12 \mathrm{H}, \mathrm{H}_{\text {alkyl }}\right) .{ }^{13} \mathrm{C}$ NMR $\left(\mathrm{CDCl}_{3}\right): \delta 154.4,146.3,137.3,132.1,128.4$, 128.2, 128.0, 127.2, 126.9, 121.6, 53.5, 50.1, 45.3, 33.6, 30.3, 29.1, 27.6, 27.3, 26.6, 25.1, 15.7, 13.8, 10.9. ${ }^{119} \mathrm{Sn} \mathrm{NMR}\left(\mathrm{CDCl}_{3}\right): \delta-38.5$. HRMS calcd for $\mathrm{C}_{27} \mathrm{H}_{45} \mathrm{~N}_{2} \mathrm{Sn}$ 517.2605 [M-Br] ${ }^{+}$; found 517.2578.

4.1.8. 1-(6-(Dibutyl(3-hydroxyprop-1-en-1-yl)stannyl)hexyl)-3ethyl-1H-imidazol-3-ium (4c). ${ }^{1} \mathrm{H}$ NMR $\left(\mathrm{CDCl}_{3}\right): \delta 10.22\left(\mathrm{~s}, 1 \mathrm{H}, \mathrm{CH}_{\mathrm{i}-}\right.$ midazolium), $7.52\left(\mathrm{~s}, 1 \mathrm{H}, \mathrm{CH}_{\text {imidazolium }}\right), 7.45\left(\mathrm{~s}, 1 \mathrm{H}, \mathrm{CH}_{\text {imidazolium }}\right)$ 5.89-5.87 (m, $\left.1 \mathrm{H}, \mathrm{CH}_{\text {alkene }}\right), 5.18-5.22\left(\mathrm{~m}, 1 \mathrm{H}, \mathrm{CH}_{\text {alkene }}\right), 4.41(\mathrm{q}$ $\left.J=7.3 \mathrm{~Hz}, 2 \mathrm{H}, \mathrm{N}-\mathrm{CH}_{2}-\mathrm{CH}_{3}\right), 4.33-4.26\left(\mathrm{~m}, 4 \mathrm{H}, \mathrm{N}-\mathrm{CH}_{2}-\mathrm{CH}_{2}\right.$ and $\left.\mathrm{CH}_{2}-\mathrm{OH}\right), 2.99(\mathrm{~s}, 1 \mathrm{H}, \mathrm{OH}), 1.94-1.86\left(\mathrm{~m}, 2 \mathrm{H}, \mathrm{N}-\mathrm{CH}_{2}-\mathrm{CH}_{2}\right), 1.58(\mathrm{t}$, $\left.J=7.3 \mathrm{~Hz}, 3 \mathrm{H}, \mathrm{N}-\mathrm{CH}_{2}-\mathrm{CH}_{3}\right), 1.55-1.41\left(\mathrm{~m}, 6 \mathrm{H}, \mathrm{H}_{\text {alkyl }}\right), 1.39-1.23(\mathrm{~m}$, $\left.10 \mathrm{H}, \mathrm{H}_{\text {alkyl }}\right), 0.96-0.84\left(\mathrm{~m}, 10 \mathrm{H}, \mathrm{H}_{\text {alkyl }}\right) .{ }^{13} \mathrm{C}$ NMR $\left(\mathrm{CDCl}_{3}\right): \delta 155.0$, 136.7, 132.0, 128.6, 122.7, 122.0, 68.9, 50.1, 45.3, 33.2, 30.0, 29.1, 27.4, 26.5, 25.5, 15.6, 13.7, 9.4. ${ }^{119} \mathrm{Sn}$ NMR $\left(\mathrm{CDCl}_{3}\right): \delta-47.4$. HRMS calcd for $\mathrm{C}_{22} \mathrm{H}_{43} \mathrm{~N}_{2} \mathrm{OSn} 471.2397[\mathrm{M}-\mathrm{Br}]^{+}$; found 471.2358.

4.1.9. (E)-1-((Dibutyl(1,2-diphenylvinyl)stannyl)methyl)-3-ethyl-1Himidazol-3-ium bromide (5a). ${ }^{1} \mathrm{H}$ NMR $\left(\mathrm{CDCl}_{3}\right): \delta 10.48\left(\mathrm{~s}, 1 \mathrm{H}, \mathrm{CH}_{\mathrm{i}-}\right.$ midazolium), 7.64 (s, $\left.1 \mathrm{H}, \mathrm{CH}_{\text {imidazolium }}\right), 7.38$ (s, 1H, $\left.\mathrm{CH}_{\text {imidazolium }}\right), 7.25$ $\left(\mathrm{m}, 2 \mathrm{H}, \mathrm{CH}_{\text {arom }}\right), 7.13-7.05\left(\mathrm{~m}, 4 \mathrm{H}, \mathrm{CH}_{\text {arom }}\right), 7.00-6.95(\mathrm{~m}, 4 \mathrm{H}$, $\left.\mathrm{CH}_{\text {arom }}\right), 6.64\left(\mathrm{~s}, 1 \mathrm{H}, \mathrm{CH}_{\text {alkene }}\right), 4.42\left(\mathrm{q}, \mathrm{J}=7.3 \mathrm{~Hz}, 2 \mathrm{H}, \mathrm{N}-\mathrm{CH}_{2}-\mathrm{CH}_{3}\right)$, $4.28\left(\mathrm{t}, J=7.5 \mathrm{~Hz}, 2 \mathrm{H}, \mathrm{N}-\mathrm{CH}_{2}-\mathrm{CH}_{2}\right), 1.85\left(\mathrm{~m}, 2 \mathrm{H}, \mathrm{N}-\mathrm{CH}_{2}-\mathrm{CH}_{2}\right), 1.58$ (t, $\left.J=7.3 \mathrm{~Hz}, 3 \mathrm{H}, \mathrm{N}-\mathrm{CH}_{2}-\mathrm{CH}_{3}\right), 1.51-1.40\left(\mathrm{~m}, 6 \mathrm{H}, \mathrm{H}_{\text {alkyl }}\right), 1.34-1.23$ $\left(\mathrm{m}, 8 \mathrm{H}, \mathrm{H}_{\text {alkyl }}\right), 0.98-0.84\left(\mathrm{~m}, 12 \mathrm{H}, \mathrm{H}_{\text {alkyl }}\right) .{ }^{13} \mathrm{C} \mathrm{NMR}\left(\mathrm{CDCl}_{3}\right): \delta 149.7$, 145.5, 138.1,136.7,137.3, 129.0, 128.5, 127.7, 126.5, 126.1, 124.9, 122.7, 121.6, 49.9, 45.1, 33.6, 30.2, 28.8, 27.1, 26.9, 26.4, 25.6, 25.6, 13.5, 9.9. ${ }^{119} \mathrm{Sn}$ NMR $\left(\mathrm{CDCl}_{3}\right): \delta-34.8$. HRMS calcd for $\mathrm{C}_{33} \mathrm{H}_{49} \mathrm{~N}_{2} \mathrm{Sn} 593.2918$ $[\mathrm{M}-\mathrm{Br}]^{+}$; found 593.2910 .

4.1.10. (E)-1-((Dibutyl(3-hydroxy-3-methylbut-1-en-1-yl)stannyl) hexyl)-3-ethyl-1H-imidazol-3-ium bromide (6a). ${ }^{1} \mathrm{H}$ NMR $\left(\mathrm{CDCl}_{3}\right)$ : $\delta 10.46\left(\mathrm{~s}, 1 \mathrm{H}, \mathrm{CH}_{\text {imidazolium }}\right), 7.69\left(\mathrm{~s}, 1 \mathrm{H}, \mathrm{CH}_{\text {imidazolium }}\right), 7.53(\mathrm{~s}, 1 \mathrm{H}$, $\left.\mathrm{CH}_{\text {imidazolium }}\right), 6.14$ (d, J=19.0 Hz, 1H, CHalkene), 6.07 (d, $J=19.0 \mathrm{~Hz}$, $\left.1 \mathrm{H}, \mathrm{CH}_{\text {alkene }}\right), 4.45$ (q, J=7.4 Hz, $\left.2 \mathrm{H}, \mathrm{N}-\mathrm{CH}_{2}-\mathrm{CH}_{3}\right), 4.34(\mathrm{t}, J=7.5 \mathrm{~Hz}$, $\left.2 \mathrm{H}, \mathrm{N}-\mathrm{CH}_{2}-\mathrm{CH}_{2}\right), 2.29(\mathrm{~s}, 1 \mathrm{H}, \mathrm{OH}), 1.92\left(\mathrm{~m}, 2 \mathrm{H}, \mathrm{N}-\mathrm{CH}_{2}-\mathrm{CH}_{2}\right), 1.60$ (t, J=7.4 Hz, 3H, N- $\left.\mathrm{CH}_{2}-\mathrm{CH}_{3}\right), 1.56-1.24\left(\mathrm{~m}, 20 \mathrm{H}, \mathrm{H}_{\text {alkyl }}\right), 0.95(\mathrm{~m}$, $\left.12 \mathrm{H}, \mathrm{H}_{\mathrm{alkyl}}\right) .{ }^{13} \mathrm{C} \mathrm{NMR}\left(\mathrm{CDCl}_{3}\right): \delta 165.7,155.1,136.7,122.1,121.9,49.9$, 45.2, 32,0, 28.2, 26.8, 25.2, 24.9, 21.4, 15.7, 13.7, 9.4. ${ }^{119}$ Sn NMR $\left(\mathrm{CDCl}_{3}\right): \delta-45.9$. HRMS calcd for $\mathrm{C}_{24} \mathrm{H}_{47} \mathrm{~N}_{2} \mathrm{OSn} 499.2710[\mathrm{M}-\mathrm{Br}]^{+}$; found 499.2732 .

4.1.11. 1-(6-(Dibutyl(4-cyanostyryl)stannyl)hexyl)-3-ethyl-1H-imidazol-3-ium bromide (7c). ${ }^{1} \mathrm{H}$ NMR $\left(\mathrm{CDCl}_{3}\right): \delta 10.43\left(\mathrm{~s}, 1 \mathrm{H}, \mathrm{CH}_{\text {imidazo- }}\right.$ lium), 7.60-7.57 (m, 2H, CH arom), $7.55\left(\mathrm{~m}, 1 \mathrm{H}, \mathrm{CH}_{\text {imidazolium }}\right), 7.41(\mathrm{~m}$, $\left.1 \mathrm{H}, \mathrm{CH}_{\text {imidazolium }}\right), 7.22-7.19\left(\mathrm{~m}, 2 \mathrm{H}, \mathrm{CH}_{\text {arom }}\right), 6.02(\mathrm{~d}, J=2.2 \mathrm{~Hz}, 1 \mathrm{H}$, $\left.\mathrm{CH}_{\text {alkene }}\right), 5.53$ (d, $\left.J=2.2 \mathrm{~Hz}, 1 \mathrm{H}, \mathrm{CH}_{\text {alkene }}\right), 4.44(\mathrm{q}, J=7.3 \mathrm{~Hz}, 2 \mathrm{H}$, $\left.\mathrm{N}-\mathrm{CH}_{2}-\mathrm{CH}_{3}\right), 4.33\left(\mathrm{t}, \mathrm{J}=7.5 \mathrm{~Hz}, 2 \mathrm{H}, \mathrm{N}-\mathrm{CH}_{2}-\mathrm{CH}_{2}\right), 1.94-1.82(\mathrm{~m}, 2 \mathrm{H}$, $\left.\mathrm{N}-\mathrm{CH}_{2}-\mathrm{CH}_{2}\right), 1.56\left(\mathrm{t}, \mathrm{J}=7.3 \mathrm{~Hz}, 3 \mathrm{H}, \mathrm{N}-\mathrm{CH}_{2}-\mathrm{CH}_{3}\right), 1.52-1.23(\mathrm{~m}$, $\left.14 \mathrm{H}, \mathrm{H}_{\text {alkyl }}\right), 1.02-0.89\left(\mathrm{~m}, 6 \mathrm{H}, \mathrm{H}_{\text {alkyl }}\right), 0.86\left(\mathrm{t}, J=7.3 \mathrm{~Hz}, 6 \mathrm{H}, \mathrm{CH}_{3 \text { butyl }}\right)$. ${ }^{13} \mathrm{C}$ NMR $\left(\mathrm{CDCl}_{3}\right): \delta 153.6,151.8,136.9,132.2,129.5,126.8,121.9$, 119.2, 109.4, 50.1, 45.3, 33.7, 30.3, 29.9, 27.2, 26.6, 25.8, 15.6, 13.7, 10.3. ${ }^{119} \mathrm{Sn}$ NMR $\left(\mathrm{CDCl}_{3}\right): \delta-42.9$. HRMS calcd for $\mathrm{C}_{28} \mathrm{H}_{44} \mathrm{~N}_{3} \mathrm{Sn}$ $542.2563[\mathrm{M}-\mathrm{Br}]^{+}$; found 542.2568 .

4.1.12. (E)-1-(6-(Dibutyl(2-(pyridin-2-yl)vinyl)stannyl)hexyl)-3ethyl-1H-imidazol-3-ium bromide $(\mathbf{8 a}) .{ }^{1} \mathrm{H} \mathrm{NMR}\left(\mathrm{CDCl}_{3}\right): \delta 10.78(\mathrm{~s}$, $\left.1 \mathrm{H}, \mathrm{CH}_{\text {imidazolium }}\right), 8.58-8.48\left(\mathrm{~m}, 1 \mathrm{H}, \mathrm{CH}_{\text {pyridine }}\right), 7.72-7.60(\mathrm{~m}, 1 \mathrm{H}$, $\left.\mathrm{CH}_{\text {pyridine }}\right), \quad 7.52-7.41\left(\mathrm{~m}, 1 \mathrm{H}, \quad \mathrm{CH}_{\text {imidazolium }}\right), 7.35(\mathrm{~s}, 1 \mathrm{H}$,
$\left.\mathrm{CH}_{\text {imidazolium }}\right), 7.31$ ( $\left.\mathrm{m}, 2 \mathrm{H}, \mathrm{CH}_{\text {pyridine }}\right), 7.23-6.98$ ( $\left.\mathrm{m}, 2 \mathrm{H}, \mathrm{CH}_{\text {alkene }}\right)$, $4.42\left(\mathrm{q}, J=7.3 \mathrm{~Hz}, 2 \mathrm{H}, \mathrm{N}-\mathrm{CH}_{2}-\mathrm{CH}_{3}\right), 4.27(\mathrm{t}, J=7.5 \mathrm{~Hz}, 2 \mathrm{H}$, $\left.\mathrm{N}-\mathrm{CH}_{2}-\mathrm{CH}_{2}\right), 1.99-1.93\left(\mathrm{~m}, 2 \mathrm{H}, \mathrm{N}-\mathrm{CH}_{2}-\mathrm{CH}_{2}\right), 1.68(\mathrm{t}, J=7.3 \mathrm{~Hz}, 3 \mathrm{H}$, $\left.\mathrm{N}-\mathrm{CH}_{2}-\mathrm{CH}_{3}\right), 1.56-1.48\left(\mathrm{~m}, 5 \mathrm{H}, \mathrm{H}_{\text {alkyl }}\right), 1.40-1.27$ (m, 9H, $\mathrm{H}_{\text {alkyl }}$, 0.95-0.79 (m, $\left.12 \mathrm{H}, \mathrm{H}_{\mathrm{alkyl}}\right) .{ }^{13} \mathrm{C} \mathrm{NMR}\left(\mathrm{CDCl}_{3}\right): \delta 149.3,148.0,136.7$, 135.6, 122.2, 121.4, 121.0, 117.9, 50.2, 45.4, 33.6, 30.3, 27.4, 25.8, 25.7, 15.6, 13.8, 10.8, 9.0. ${ }^{119} \mathrm{Sn}$ NMR $\left(\mathrm{CDCl}_{3}\right): \delta-44.4$. HRMS calcd for $\mathrm{C}_{26} \mathrm{H}_{44} \mathrm{~N}_{3} \mathrm{Sn} 518.2562[\mathrm{M}-\mathrm{Br}]^{+}$; found 518.2571.

4.1.13. 1-(6-(Dibutyl(pent-1-en-1-yl)stannyl)hexyl)-3-ethyl-1H-imidazol-3-ium bromide $(\mathbf{9 c}) .{ }^{1} \mathrm{H}$ NMR $\left(\mathrm{CDCl}_{3}\right): \delta 10.37\left(\mathrm{~s}, 1 \mathrm{H}, \mathrm{CH}_{\text {imida- }}\right.$ zolium), $7.63\left(\mathrm{~m}, 1 \mathrm{H}, \mathrm{CH}_{\text {imidazolium }}\right), 7.41\left(\mathrm{~m}, 1 \mathrm{H}, \mathrm{CH}_{\text {imidazolium }}\right)$, 5.76-5.96 (m, $\left.2 \mathrm{H}, \mathrm{CH}_{\text {alkene }}\right), 4.42\left(\mathrm{q}, J=7.3 \mathrm{~Hz}, 2 \mathrm{H}, \mathrm{N}-\mathrm{CH}_{2}-\mathrm{CH}_{3}\right)$, $4.30\left(\mathrm{t}, J=7.5 \mathrm{~Hz}, 2 \mathrm{H}, \mathrm{N}-\mathrm{CH}_{2}-\mathrm{CH}_{2}\right), 2.07\left(\mathrm{~m}, 2 \mathrm{H},=\mathrm{C}-\mathrm{CH}_{2}\right), 1.88(\mathrm{~m}$, $\left.2 \mathrm{H}, \mathrm{N}-\mathrm{CH}_{2}-\mathrm{CH}_{2}\right), 1.58\left(\mathrm{t}, \mathrm{J}=7.3 \mathrm{~Hz}, 3 \mathrm{H}, \mathrm{N}-\mathrm{CH}_{2}-\mathrm{CH}_{3}\right), 1.51-1.23(\mathrm{~m}$, $\left.16 \mathrm{H}, \mathrm{H}_{\text {alkyl }}\right), 0.88-0.79\left(\mathrm{~m}, 15 \mathrm{H}, \mathrm{H}_{\text {alkyl }}\right) .{ }^{13} \mathrm{C} \mathrm{NMR}\left(\mathrm{CDCl}_{3}\right): \delta 149.8$, 136.9, 132.0, 128.5, 122.0, 45.3, 39.9, 29.0, 27.3, 25.9, 22.0, 13.7, 9.4. ${ }^{119} \mathrm{Sn}$ NMR $\left(\mathrm{CDCl}_{3}\right): \delta-50.7$. HRMS calcd for $\mathrm{C}_{24} \mathrm{H}_{47} \mathrm{~N}_{2} \mathrm{Sn} 483.2766$ $[\mathrm{M}-\mathrm{Br}]^{+}$; found 483.2798 .

4.1.14. (E)-1-((Dibutyl(3,3-dimethylbut-1-en-1-yl)stannyl)hexyl)-3ethyl-1H-imidazol-3-ium bromide (10a). ${ }^{1} \mathrm{H} \mathrm{NMR}\left(\mathrm{CDCl}_{3}\right): \delta 10.67$ (s, $\left.1 \mathrm{H}, \mathrm{CH}_{\text {imidazolium }}\right), 7.34\left(\mathrm{~s}, 1 \mathrm{H}, \mathrm{CH}_{\text {imidazolium }}\right), 7.24\left(\mathrm{~s}, 1 \mathrm{H}, \mathrm{CH}_{\text {imidazo- }}\right.$ lium), 5.95 (d, $\left.J=19.3 \mathrm{~Hz}, 1 \mathrm{H}, \mathrm{CH}_{\text {alkene }}\right), 5.75$ (d, $J=19.3 \mathrm{~Hz}, 1 \mathrm{H}, \mathrm{CH}_{\mathrm{al}-}$ kene), 4.45 (q, $\left.J=7.3 \mathrm{~Hz}, 2 \mathrm{H}, \mathrm{N}-\mathrm{CH}_{2}-\mathrm{CH}_{3}\right), 4.35(\mathrm{t}, J=7.5 \mathrm{~Hz}, 2 \mathrm{H}$, $\left.\mathrm{N}-\mathrm{CH}_{2}-\mathrm{CH}_{2}\right), 2.01-1.84\left(\mathrm{~m}, 2 \mathrm{H}, \mathrm{N}-\mathrm{CH}_{2}-\mathrm{CH}_{2}\right), 1.79-1.65(\mathrm{~m}, 6 \mathrm{H}$, $\left.\mathrm{H}_{\text {alkyl }}\right), 1.62\left(\mathrm{t}, J=7.3 \mathrm{~Hz}, 3 \mathrm{H}, \mathrm{N}-\mathrm{CH}_{2}-\mathrm{CH}_{3}\right), 1.52-1.22\left(\mathrm{~m}, 14 \mathrm{H}, \mathrm{H}_{\text {alkyl }}\right)$, $1.01-0.80\left(\mathrm{~m}, 15 \mathrm{H}, \mathrm{H}_{\mathrm{alkyl}}\right) .{ }^{13} \mathrm{C} \mathrm{NMR}\left(\mathrm{CDCl}_{3}\right): \delta 159.9,136.1,130.7$, 121.9, 121.7, 119.9, 49.8, 45.2, 32, 29.6, 28.2, 26.8, 25.2, 24.9, 21.9, $21.4,15.5 .{ }^{119} \mathrm{Sn}$ NMR $\left(\mathrm{CDCl}_{3}\right): \delta-46.4$. HRMS calcd for $\mathrm{C}_{25} \mathrm{H}_{49} \mathrm{~N}_{2} \mathrm{Sn}$ : 497.2918 [M-Br] $]^{+}$; found 497.2964.

4.1.15. 1-\{6-[Dibutylchlorostannyl]hexyl\}-3-ethyl-1H-imidazol-3ium tetrafluoroborate (11). ${ }^{1} \mathrm{H} \mathrm{NMR}\left(\mathrm{CDCl}_{3}\right): \delta 8.85\left(\mathrm{~s}, 1 \mathrm{H}, \mathrm{CH}_{\text {imida- }}\right.$ zolium), 7.41 (s, $\left.1 \mathrm{H}, \mathrm{CH}_{\text {imidazolium }}\right), 7.37$ (s, $1 \mathrm{H}, \mathrm{CH}_{\text {imidazolium }}$ ), 4.27 (q, $\left.J=7.2 \mathrm{~Hz}, 2 \mathrm{H}, \mathrm{N}-\mathrm{CH}_{2}-\mathrm{CH}_{3}\right), 4.20\left(\mathrm{t}, J=7.2 \mathrm{~Hz}, 2 \mathrm{H}, \mathrm{N}-\mathrm{CH}_{2}-\mathrm{CH}_{2}\right), 1.89$ $\left(\mathrm{m}, 2 \mathrm{H}, \mathrm{N}-\mathrm{CH}_{2}-\mathrm{CH}_{2}\right), 1.76-1.52\left(\mathrm{~m}, 9 \mathrm{H}, \mathrm{H}_{\text {alkyl }}\right), 1.46-1.22(\mathrm{~m}, 14 \mathrm{H}$, $\left.\mathrm{H}_{\text {alkyl }}\right), 0.91\left(\mathrm{t}, J=7.3 \mathrm{~Hz}, 6 \mathrm{H}, \mathrm{CH}_{3 \text { butyl }}\right) .{ }^{13} \mathrm{C}$ NMR $\left(\mathrm{CDCl}_{3}\right): \delta 135.1$, 122.2, 121.9, 50.1, 45.4, 31.6, 29.6, 28.2, 26.4, 25.1, 24.3, 21.7, 21.2, 15.1, 13.7. ${ }^{119} \mathrm{Sn} \mathrm{NMR}\left(\mathrm{CDCl}_{3}\right)$ : $\delta$ 149.7. HRMS calcd for $\mathrm{C}_{19} \mathrm{H}_{38} \mathrm{~N}_{2} \mathrm{Sn}$ $449.1746\left[\mathrm{M}-\mathrm{BF}_{4}\right]^{+}$; found 449.1732 .

4.1.16. 1-\{6-[Dibutylchlorostannyl]hexyl\}-3-ethyl-1H-imidazol-3ium hexafluoroantimonate (12). ${ }^{1} \mathrm{H}$ NMR $\left(\mathrm{CDCl}_{3}\right): \delta 8.63(\mathrm{~s}, 1 \mathrm{H}$, $\mathrm{CH}_{\text {imidazolium }}$ ), 7.35 (s, 2H, $\left.\mathrm{CH}_{\text {imidazolium }}\right), 4.21$ (q, $J=7.6,2 \mathrm{H}$, $\left.\mathrm{N}-\mathrm{CH}_{2}-\mathrm{CH}_{3}\right), 4.19\left(\mathrm{t}, \mathrm{J}=7.2 \mathrm{~Hz}, 2 \mathrm{H}, \mathrm{N}-\mathrm{CH}_{2}-\mathrm{CH}_{2}\right), 2.01-1.83(\mathrm{~m}, 2 \mathrm{H}$, $\left.\mathrm{N}-\mathrm{CH}_{2}-\mathrm{CH}_{2}\right), 1.8-1.65\left(\mathrm{~m}, 6 \mathrm{H}, \mathrm{H}_{\text {alkyl }}\right), 1.62(\mathrm{t}, J=7.6 \mathrm{~Hz}, 3 \mathrm{H}$, $\left.\mathrm{N}-\mathrm{CH}_{2}-\mathrm{CH}_{3}\right), 1.50-1.30\left(\mathrm{~m}, 14 \mathrm{H}, \mathrm{H}_{\text {alkyl }}\right), 0.9(\mathrm{t}, J=7.2 \mathrm{~Hz}, 6 \mathrm{H}$, $\left.\mathrm{CH}_{3 \text { butyl }}\right) .{ }^{13} \mathrm{C}$ NMR $\left(\mathrm{CDCl}_{3}\right): \delta 135.0,120.9,120.1,50.1,45.2,32,29.2$, 29.1, 26.8, 25.1, 17.6, 15.1, 13.6. ${ }^{119} \mathrm{Sn} \mathrm{NMR}\left(\mathrm{CDCl}_{3}\right)$ : $\delta$ 156.0. HRMS calcd for $\mathrm{C}_{19} \mathrm{H}_{38} \mathrm{~N}_{2} \mathrm{Sn} 449.1746\left[\mathrm{M}-\mathrm{SbF}_{6}\right]^{+}$; found 449.1767 .

4.1.17. (E)-1-(6-(Dibutyl(3-hydroxy-3-methylbut-1-en-1-yl)stannyl) hexyl)-3-ethyl-1H-imidazol-3-ium tetrafluoroborate (13). ${ }^{1} \mathrm{H}$ NMR $\left(\mathrm{CDCl}_{3}\right): \delta 9.08\left(\mathrm{~s}, 1 \mathrm{H}, \mathrm{CH}_{\text {imidazolium }}\right), 7.37-7.31\left(\mathrm{~m}, 2 \mathrm{H}, \mathrm{CH}_{\text {imidazolium }}\right)$ 6.39-6.10 (m, 1H, CHalkene), 5.94-5.72 (m, 1H, CHalkene), $4.32(\mathrm{q}$, $\left.J=7.3 \mathrm{~Hz}, 2 \mathrm{H}, \mathrm{N}-\mathrm{CH}_{2}-\mathrm{CH}_{3}\right), 4.24\left(\mathrm{t}, J=7.5 \mathrm{~Hz}, 2 \mathrm{H}, \mathrm{N}-\mathrm{CH}_{2}-\mathrm{CH}_{2}\right)$, 1.98-1.86 (m, 2H, N- $\left.\mathrm{CH}_{2}-\mathrm{CH}_{2}\right), 1.78-1.20\left(\mathrm{~m}, 30 \mathrm{H}, \mathrm{H}_{\text {alkyl }}\right), 0.95(\mathrm{t}$, $\left.J=7.3 \mathrm{~Hz}, 6 \mathrm{H}, \mathrm{CH}_{3 \text { butyl }}\right){ }^{13} \mathrm{C}$ NMR (acetone- $\left.d_{6}\right) \delta 158.2,136.6,123.5$, 123.2, 121.4, 72.1, 50.5, 45.7, 34.3, 30.4, 27.8, 26.4, 19.5, 15.6, 14.0, 9.9. ${ }^{119}$ Sn NMR (acetone- $d_{6}$ ): $\delta-46.8$. HRMS calcd for $\mathrm{C}_{24} \mathrm{H}_{47} \mathrm{~N}_{2} \mathrm{OSn}$ 499.2710 [M-BF $]^{+}$; found 499.2747.

4.1.18. (E)-1-(6-(Dibutyl(3-hydroxy-3-methylbut-1-en-1-yl)stannyl) hexyl)-3-ethyl-1H-imidazol-3-ium hexafluoroantimonate $(\mathbf{1 4}) .{ }^{1} \mathrm{H}$ NMR $\left(\mathrm{CDCl}_{3}\right): \delta 9.75\left(\mathrm{~s}, 1 \mathrm{H}, \mathrm{CH}_{\text {imidazolium }}\right), 7.55\left(\mathrm{~s}, 1 \mathrm{H}, \mathrm{CH}_{\text {imidazolium }}\right)$, 
$7.42\left(\mathrm{~s}, 1 \mathrm{H}, \mathrm{CH}_{\text {imidazolium }}\right), 5.92$ (d, $\left.J=19.0 \mathrm{~Hz}, 1 \mathrm{H}\right), 5.71(\mathrm{~d}, J=19.0 \mathrm{~Hz}$, $\left.1 \mathrm{H}, \mathrm{CH}_{\text {alkene }}\right), 4.33\left(\mathrm{q}, J=7.3 \mathrm{~Hz}, 2 \mathrm{H}, \mathrm{N}-\mathrm{CH}_{2}-\mathrm{CH}_{3}\right), 4.23(\mathrm{t}, J=7.5 \mathrm{~Hz}$, $\left.2 \mathrm{H}, \mathrm{N}-\mathrm{CH}_{2}-\mathrm{CH}_{2}\right), 1.91-1.71\left(\mathrm{~m}, 2 \mathrm{H}, \mathrm{N}-\mathrm{CH}_{2}-\mathrm{CH}_{2}\right), 1.51(\mathrm{t}, J=7.3 \mathrm{~Hz}$, $\left.3 \mathrm{H}, \mathrm{N}-\mathrm{CH}_{2}-\mathrm{CH}_{3}\right), 1.46-1.18\left(\mathrm{~m}, 21 \mathrm{H}, \mathrm{H}_{\text {alkyl }}\right), 0.81\left(\mathrm{~m}, 12 \mathrm{H}, \mathrm{H}_{\text {alkyl }}\right)$. ${ }^{13} \mathrm{C}$ NMR $\left(\mathrm{CDCl}_{3}\right): \delta 125.0,122.2,121.3,50.3,45.6,32.6,29.3,27.9$, $26.8,25.4,17.6,15.1,13.7 .{ }^{119} \mathrm{Sn}$ NMR $\left(\mathrm{CDCl}_{3}\right): \delta-45.8$. HRMS calcd for $\mathrm{C}_{24} \mathrm{H}_{47} \mathrm{~N}_{2} \mathrm{OSn} 499.2710\left[\mathrm{M}-\mathrm{SbF}_{6}\right]^{+}$; found 499.2659.

\section{Acknowledgements}

We thank CNRS (Centre National de la Recherche Scientifique), the Université d'Angers and the Agence Nationale de la Recherche. We also thank Amélie Durand and Patricia Gangnery for technical support.

\section{References and notes}

1. (a) Gerlach, M.; Jördens, F.; Kuhn, H.; Neumann, W. P. J. Org. Chem. 1991, 56, 5971; (b) Dumarin, G.; Ruel, G.; Kharboutli, J.; Delmond, B.; Connil, M.-F.; Jousseaume, B.; Pereyre, M. Synlett 1994, 952; (c) Enholm, E. J.; Schulte, J. P., II. Org. Lett. 1999, 1, 1275; (d) Zhu, X.; Blough, B. E.; Carroll, F. I. Tetrahedron Lett. 2000, 41, 9219; (e) Cossy, J.; Rasamison, C.; Gomez Pardo, D. J. Org. Chem. 2001, 66, 7195; (f) Chrétien, J.-M.; Zammattio, F.; Le Grognec, E.; Paris, M.; Cahingt, B.; Montavon;, G.; Quintard, J.-P. J. Org. Chem. 2005, 70, 2870; (g) Chrétien, J. M.; Mallinger, A.; Zammattio, F.; Le Grognec, E.; Paris, M.; Montavon, G.; Quintard, J.-P. Tetrahedron Lett. 2007, 48, 1781; (h) Carrera, N.; Gutierrez, E.; Benavente, R.; Mar Villavieja, M.; Albeniz, A. C.; Espinet, P. Chem.-Eur. J. 2008, 14, 10141; (i)
Carrera, N.; Salinas-Castillo, A.; Albeniz, A. C.; Espinet, P.; Mallavia, R. J. Organomet. Chem. 2011, 696, 3316.

2. Poupon, J. C.; Marcoux, D.; Cloarec, J. M.; Charette, A. B. Org. Lett. 2007, 9, 3591.

3. (a) Maleczka, R. E.; Gallagher, W. P.; Terstiege, I. J. Am. Chem. Soc. 2000, 122, 384; (b) Gallagher, W. P.; Terstiege, I.; Maleczka, R. E. J. Am. Chem. Soc. 2001, 123, 3194; (c) Maleczka, R. E.; Gallagher, W. P. Org. Lett. 2001, 3, 4173; (d) Hernán, A. G.; Guillot, V.; Kuvshinov, A.; Kilburn, J. D. Tetrahedron Lett. 2003, 44, 8601.

4. (a) Olofsson, K.; Kim, S.-Y.; Larhed, M.; Curran, D. P.; Halberg, A. J. Org. Chem. 1999, 64, 4539; (b) Fouquet, E.; Pereyre, M.; Rodriguez, A. L. J. Org. Chem. 1997, 62, 5242; (c) Fouquet, E.; Pereyre, M.; Roulet, T. J. Chem. Soc., Chem. Commun. 1995, 2387.

5. (a) Vitz, J.; Mac, D. H.; Legoupy, S. Green Chem. 2007, 9, 431; (b) Pham, P. D.; Vitz, J.: Chamignon, C.; Martel, A.; Legoupy, S. Eur. J. Org. Chem. 2009, 3249; (c) Pham, P. D.; Legoupy, S. Tetrahedron Lett. 2009, 50, 3780; (d) Pham, P. D.; Bertus, P.; Legoupy, S. Chem. Commun. 2009, 6207; (e) Louaisil, N.; Pham, P. D.; Boeda, F.; Djibril, F.; Castanet, A.-S.; Legoupy, S. Eur. J. Org. Chem. 2011, 143.

6. (a) Beletskaya, I; Moberg, C. Chem Rev 1999 99, 3435; (b) Smith, N. D.; Mancuso, J.; Lautens, M. Chem. Rev. 2000, 100, 3257; (c) Miura, K.; Wang, D.; Matsumoto, Y.; Hosomi, A. Org. Lett. 2005, 7, 503; (d) Darwish, A.; Lang, A.; Kim, T.; Chong, J. M. Org. Lett. 2008, 10, 861; (e) Hamze, A.; Veau, D.; Provot, O.; Brion, J.-D.; Alami, M. J. Org. Chem. 2009, 74, 1337.

7. Cai, M.; Wang, Y.; Hao, W. Eur. J. Org. Chem. 2008, 2983.

8. Fernandes, A. M.; Rocha, M. A. A.; Freire, M. G.; Marrucho, I. M.; Coutinho, J. A. P.; Santos, L. M. N. B. F. J. Phys. Chem. B 2011, 115, 4033.

9. Maleczka, R. E., Jr.; Lavis, J. M.; Clark, D. H.; Gallagher, W. P. Org. Lett. 2000, 2, 3655.

10. Sore, H. F.; Boehner, C. M.; Laraia, L.; Logoteta, P.; Prestinari, C.; Scott, M.; Williams, K.; Galloway, W. R. J. D. Org. Biomol. Chem. 2011, 9, 504.

11. Nakao, Y.; Imanaka, H.; Chen, J.; Yada, A.; Hiyama, T. J. Organomet. Chem. 2007, 692,585

12. Sauza, A.; Morales-Serna, J. A.; García-Molina, M.; Gaviño, R.; Cárdenas, J. Synthesis 2012, 272. 